\author{
Marquette University \\ e-Publications@Marquette
}

\title{
8-2013
}

\section{Young's Modulus and Volume Porosity Relationships for Additive Manufacturing Applications}

John Anthony Choren

Marquette University

Stephen M. Heinrich

Marquette University, stephen.heinrich@marquette.edu

M. Barbara Silver-Thorn

Marquette University, barbara.silver-thorn@marquette.edu

Follow this and additional works at: https://epublications.marquette.edu/civengin_fac

Part of the Biomedical Engineering and Bioengineering Commons, and the Civil and Environmental

Engineering Commons

\section{Recommended Citation}

Choren, John Anthony; Heinrich, Stephen M.; and Silver-Thorn, M. Barbara, "Young's Modulus and Volume Porosity Relationships for Additive Manufacturing Applications" (2013). Civil and Environmental

Engineering Faculty Research and Publications. 16.

https://epublications.marquette.edu/civengin_fac/16 
Marquette University

e-Publications@Marquette

\section{Civil and Environmental Engineering Faculty Research and Publications/College of Engineering}

This paper is NOT THE PUBLISHED VERSION; but the author's final, peer-reviewed manuscript. The published version may be accessed by following the link in the citation below.

Journal of Materials Science, Vol. 18 (April 02, 2013): 5103-5112. DOI. This article is @ Springer and permission has been granted for this version to appear in e-Publications@Marquette. Springer does not grant permission for this article to be further copied/distributed or hosted elsewhere without the express permission from Springer.

\section{Young's Modulus and Volume Porosity Relationships for Additive Manufacturing Applications}

\section{J. A. Choren}

Milwaukee School of Engineering, Milwaukee, WI

Department of Biomedical Engineering, Marquette University, Milwaukee, WI

Triad Engineering LLC, Oconomowoc, WI

S. M. Heinrich

Department of Civil, Construction and Environmental Engineering, Marquette University, Milwaukee, WI

M. B. Silver-Thorn

Department of Biomedical Engineering, Marquette University, Milwaukee, WI

\section{Abstract}

Recent advancements in additive manufacturing (or rapid prototyping) technologies allow the fabrication of end-use components with defined porous structures. For example, one area of particular interest is the 
potential to modify the flexibility (bending stiffness) of orthopedic implants through the use of engineered porosity (i.e., design and placement of pores) and subsequent fabrication of the implant using additive manufacturing processes. However, applications of engineered porosity require the ability to accurately predict mechanical properties from knowledge or characterization of the pore structure and the existence of robust equations characterizing the property-porosity relationships. As Young's modulus can be altered by variations in pore shape as well as pore distribution, numerous semi-analytical and theoretical relationships have been proposed to describe the dependence of mechanical properties on porosity. However, the utility and physical meaning of many of these relationships is often unclear as most theoretical models are based on some idealized physical microstructure, and the resulting correlations often cannot be applied to real materials and practical applications. This review summarizes the evolution and development of relationships for the effective Young's modulus of a porous material and concludes that verifiable equations yielding consistently reproducible results tied to specific pore structures do not yet exist. Further research is needed to develop and validate predictive equations for the effective Young's modulus over a volume porosity range of 20-50 \%, the range of interest over which existing equations, whether based on effective medium theories or empirical results, demonstrate the largest disparity and offers the greatest opportunity for beneficial modification of bending stiffness in orthopedic applications using currently available additive manufacturing techniques.

\section{Introduction}

Research into the mechanical properties of porous materials has focused on the characterization of a particular material or methodology to interpret the observed performance of porous media. While this research has intrinsic value, the need to apply this research to the prediction of mechanical properties for the purpose of design has not received the same attention.

Most theoretical equations for calculating effective moduli have been derived by treating a representative volume of a porous solid as a special case of two-phase materials, evaluating them mathematically or empirically, and extending the conclusions to the porous solid [1]. Exact dilute-limit formulas may be derived by evaluating the effect of a single pore, small enough to have no effect on the other pores within the matrix, on the moduli of the porous material. These exact dilute-limit relationships, however, have limited applicability to characterization of elastic moduli of real composites/porous solids that violate the requisite theoretical conditions (e.g., non-interaction among the perturbing stress fields due to the individual pores). As such, effective medium theories have been developed to extend the stress and strain distributions of the isolated single-pore solutions to account for interactions between the inclusion phases [2]. Other approaches rely on discrete sets of empirical data, quantifying relationships based on regression of experimental results for an assumed inherent porosity range.

O'Kelly et al. [3] characterize the theoretical approaches into either composite theory, which assumes a twophase material, with one phase having zero stiffness, or cellular solids and minimum solid area (MSA) approaches, which assume a single phase permeated with voids. Herakovich and Baxter [4] take a broader view and observe that there are two fundamentally different approaches to the study of porous media: those of the mechanics community and those of the materials community. The mechanics community has tended to consider a specific pore shape (often spherical) and then develop analytical solutions for mechanical properties as a function of pore volume fraction. In contrast, the materials community has tended to obtain experimental results for mechanical properties as a function of volume porosity and then determine the "best-fit" curve relating the properties to the pore geometry or method of fabrication. These different approaches yield divergent results and, in some instances, formulas which are in direct contradiction [5].

Recent developments in additive manufacturing facilitate the creation of structures with a predictable and precisely defined porosity in terms of pore size, shape, orientation, and arrangement. Application of this 
technology to specific design requirements requires robust predictive models that include these variables in the characterization of porous media. Rather than present existing mechanical property-porosity relationships in terms of the differing perspectives of the mechanics and materials communities as noted by Herakovich and Baxter [4], this review summarizes the relationships posed by investigators in both communities in terms of the equation type (linear, power, exponential, or other). Details regarding the implicit assumptions and valid porosity range, as relevant to future engineered porosity applications in additive manufacturing, are summarized.

\section{Linear equations}

Dewey [6] derived dilute-limit expressions for the elastic constants of materials containing non-rigid fillers from consideration of a single sphere (radius $=R$ ) within an isotropic medium subjected to a displacement at a large distance $(\gg R$ ) from the sphere. Accordingly, these expressions are valid for "very dilute suspensions" for which surface effects may be ignored. Dewey's model was derived to include the effects of gas pressure within pores of elastomeric materials. Although algebraic errors and inconsistencies in the evaluation of bulk and shear moduli in Dewey's analysis preclude its accurate use as published [7], the basic form of the equation for Young's modulus appears correct:

$E_{\mathrm{p}}=E_{0}(1-a P)(1)$

where $E_{\mathrm{p}}$ is Young's modulus of the porous body, $E_{0}$ is the modulus of a non-porous body of the same material, $a$ is a constant dependent on Poisson's ratio of the matrix material, and $P$ is the volume porosity.

Fryxell and Chandler [8] compared their experimental results for sintered beryllium oxide and found that the linear equation (1) "describes the data well enough for most practical purposes" for a volume porosity less than $17 \%$. Hasselman and Fulrath [9] demonstrated the validity of the Dewey equation up to $2.5 \%$ porosity for glass with spherical voids. Their theoretical value for the constant $a$ as a function of $v_{0}$, the Poisson's ratio of the nonporous material, was based on prior analyses of an isolated spherical void within an elastic solid [5, 10] (i.e., the exact dilute-limit result for a statistically isotropic distribution of spherical voids) and takes the form:

$a=3\left(9+5 v_{0}\right)\left(1-v_{0}\right) /\left[2\left(7-5 v_{0}\right)\right](2)$

The results noted by Hasselman and Fulrath showed "good [visual] agreement between theory and experiment." Rossi [11] noted that the coefficient $a$ approximates the stress concentration about a spherical cavity for compressive and shear stress states and is solely a function of the Poisson's ratio of the matrix material. He expanded the theoretical applicability of the linear equation to include oblate and prolate spheroids but cautioned that his approach is "nonrigorous and is intended only as an engineering solution to the problem" of predicting the elastic moduli of porous constructs.

\section{Power relationships}

Bert [12] expanded Rossi's work to include stress concentration factors for different void geometries and added an exponent for the maximum porosity possible, $Q$, given a specific pore arrangement:

$E_{\mathrm{p}}=E_{0}[1-(P / Q)]^{K Q}(3)$

where

$K=0.75+(1.25 b / c)(4)$ 
In this equation, $c$ is the void length parallel to the loading direction and $b$ is the void width perpendicular to the loading direction. Although empirical results are not provided, Bert maintains that Eq. (3) is more accurate than earlier equations for solids with less than $20 \%$ volume porosity.

Like Dewey, Mackenzie [10] also studied the elastic properties of porous solids, assuming that the solid material is homogeneous and isotropic with isolated voids of different sizes distributed randomly throughout the volume of the material. He states that because voids have a comparatively large effect on compressibility and only a small effect on the shear modulus, his results can be extended to different void shapes and sizes, provided that extremes are excluded. Per Chung [13] and Wagh et al. [14], Mackenzie's semi-empirical equation has the form:

$E_{\mathrm{p}}=E_{0}\left(1-d P+g P^{2}\right)(5)$

where the "slope constants" $d$ and $g$ "depend upon the shape of the pores, assuming that all of the pores are closed pores" [13]. As Mackenzie's theoretical derivation assumed non-interaction between spherical voids and included both the shear and bulk moduli, it is expected that Eq. (5) can be interpreted as a power series in $P$ which would yield the dilute-limit solution if truncated after the linear term. Mackenzie adds that "it is not clear how the numerical constants [in Eq. (5)]... will depend on the particular shape [of void] chosen... but it seems likely that the[se] formulae ... will give the correct order of magnitude in most cases." [10].

Experimental results of Spinner et al. [15] confirmed the validity Eq. (5) for polycrystalline thoria with volume porosity up to $40 \%$. Spinner et al. also noted that although the Mackenzie equation includes a second-order term, at low porosities this relationship is equivalent to the dilute-limit solution of Hashin [5] as zero porosity is approached.

Although he did not investigate the change in elastic moduli, Bal'shin [16] concluded that the strength of a brittle porous specimen, such as sintered copper, can be described with an equation of the form:

$S_{\mathrm{p}}=S_{0}(1-P)^{h}(6)$

where $S_{\mathrm{p}}$ is the ultimate tensile strength of the porous body, $S_{0}$ is the corresponding strength of the non-porous material, and $h$ is an empirical constant. Bal'shin noted that the value of the constant $h$ ranged from 3 to 6 depending on the "characteristics" of the original material and the time and temperature of sintering, but did not include data on the variation in the value of $h$, as pointed out by Knudsen [17]. McAdam [18] derived an equation similar in form to Eq. (6) for the Young's modulus of sintered ferrous alloys. For porosities up to $40 \%$, his empirical data indicated that the Young's modulus could be described by an equation of the form:

$E_{\mathrm{p}}=E_{0}(1-j P)^{i}(7)$

with the empirically determined exponents $i=3.4$ and $j=1$.

Phani and collaborators [19-23] also compared empirical results for porous materials, including thoria, uranium dioxide, gypsum, alumina, and silicon nitride, to the value of Young's modulus predicted by Eq. (7), concluding that the value of $i$ "possibly lies between 2 and 3 for a relatively ordered and less open pore structure," and is valid for porosity less than $40 \%$ [21]. The constant $j$ is the reciprocal of the critical porosity, i.e., the porosity at which the structure becomes unstable and Young's modulus falls to zero. The variability in the value of $i$ versus that of McAdam in Eq. (7) is attributed to inherent differences in the nature of the porosity [22]. The value of $i$ increases as the pores depart from being spherical and become more interconnected and it depends on "pore geometry, grain size and the interconnection of grains" [19]. Wagh et al. [14, 24] confirm these findings for ceramics, adding that the exponent $i$ depends on the "tortuosity of the [pore] structure." Maitra and Phani [25] confirmed and extended the earlier work of Phani and concluded that the value of $i$ is 2 for closed spherical 
pores and 4 for random orientation of pores; $i$ fell into a range of 3-4 in their evaluation of 12 polycrystalline materials.

Others have correlated the value of the exponent $i$ to the type and extent of stress concentrations that develop around pores [26]. Ji et al. [27] proposed an equation similar to (7) to describe the mechanical properties of porous materials provided that the value of the exponent, which depends on the "geometrical shape, spatial arrangement, orientation and size distribution of pores," can be determined from experimental data.

Accordingly, this limits the predictive capability of this equation as it relies on the existence of previous empirical determinations. In most cases, these data are graphically presented and values for $i$ are fit visually.

\section{Exponential equations}

Ryshkewitch [28] showed that strength varies logarithmically with porosity in sintered alumina and offered one of the most widely used equations to describe the effects of porosity on mechanical properties [22]. Duckworth [29] generalized the Ryshkewitch relationship as:

$S_{\mathrm{p}}=S_{0} \exp (-k P)(8)$

where $k$ is a material-dependent constant and other variables are as defined previously. Duckworth noted that $k$ "has a value of about 7 for all experimental data on hand... [it] also appears to be independent of pore size," but this equation is reportedly valid only for porosity up to $50 \%$. Knudsen [17] noted that the Duckworth Eq. (8) is "very similar to the relation proposed by Bal'shin" (6) and that since $k \approx 1.33 h$, the results of Eqs. (6) and (8) can be made to approximate each other with slight alterations in the ratio of $k$ to $h$. Knudsen assumed that strength was proportional to the contact or load-bearing area for different grain packing arrangements and showed that strength could be expressed by this exponential relationship with values of $k$ ranging from 6 to 9 for different structures $[7,17]$. The analysis by Knudsen became the basis for later work by Rice [30, 31] which yields an analogous exponential relationship, based on the MSA, to predict Young's modulus.

Spriggs [32] extended the Duckworth Eq. (8) characterizing the effect of porosity on strength to the modulus of elasticity for aluminum oxide with porosities up to $37 \%$ :

$E_{\mathrm{p}}=E_{0} \exp (-m P)(9)$

and presented supporting data for $m$ ranging from 2.7 to 4.3; these results are consistent with later work by Knudsen [33]. While deviation from calculated values for Young's modulus became significant for porosity greater than $17 \%$, Spriggs demonstrated that the constant $m$ is dependent on material processing techniques and "perhaps the type of materials and method of modulus measurement" [32].

The above studies suggest that the effective moduli, pore shape, and volume fraction, as well as fabrication method are coupled [4]. However, Eq. (9) does not satisfy the boundary condition that $E_{\mathrm{p}}$ goes to zero as $P$ approaches 1 (i.e., the matrix material is completely displaced by the voids in the material), demonstrating that these empirically developed equations are valid for a limited porosity range. Hasselman [34] maintains that "not satisfying the boundary conditions will affect the actual values of $E_{\mathrm{p}}$ and $m$ obtained from the experimental data" and thus "any conclusions based on the result obtained from the Spriggs equation cannot be regarded as entirely valid." Work by Phani et al. [23] confirmed that Eq. (9) shows large disagreement with experimental data for porosities greater than $60 \%$. However, Herakovich and Baxter [4] acknowledged the limited applicability of this Eq. (9) in noting that this relationship provides a "good approximation for effective properties up to $40 \%$ porosity."

Due to these boundary condition issues, Hasselman [34] proposed a form of a modulus-porosity relationship motivated by theoretical work done by Hashin [5] on moduli bounds for two-phase heterogeneous materials, 
setting the properties of the second phase to zero. If voids are considered to be the second phase, Hashin's general equation for the upper bound (the lower bound is zero for the case of voids) can be rewritten in simplified form as:

$E_{\mathrm{p}}=E_{0}(1+[A P /(1-A P-P)])(10)$

Hasselman proposed that the constant $A$ be selected based on experimental data. Hasselman states that the advantage of this alternative equation is that different values of the constant $A$ can be selected to approximate the behavior over different porosity intervals.

Wang [35] confirmed that the Hashin-Hasselman Eq. (10) is valid for isolated closed pores up to about $20 \%$ porosity. To extend its applicability, Wang [36] proposed a variation of the Spriggs relationship (9):

$E_{\mathrm{p}}=E_{0} \exp \left[-\left(n P+q P^{2}\right)\right](11)$

where $n$ and $q$ are "nonnegative numbers dictated by the shape of the theoretical curves." Wang presents data that suggest that this relationship is applicable to both open and closed pores up to 30 \% porosity. Panakkal et al. [37] confirmed the validity of this equation for predicting Young's modulus of sintered iron up to $22 \%$ porosity. For higher porosities, Wang [36] suggested inclusion of additional higher order terms in the exponent. Phani [19] notes that the use of such equations becomes cumbersome and the material constants lose their potential physical significance. Because neither the Spriggs (9) nor the Wang (11) equation satisfy the condition that $E_{\mathrm{p}}$ goes to zero as $P$ approaches 1 , use of these equations to evaluate Young's modulus by extrapolating fitted experimental data can result in discrepancies of approximately one order of magnitude between the predicted and observed values [25].

While Rice [7] acknowledges the mathematical limitations inherent in the Spriggs Eq. (9) noted by Hasselman and others, he adds that in practice inhomogeneous pore distributions, unusual pore structures, or particle packing arrangements with a critical porosity (i.e., the transition from isolated and closed to an open and interconnected porous structure) can result in complete loss of strength as $P$ approaches a critical value of porosity, usually well below 1 . Thus, the fact that the Spriggs Eq. (9) does not satisfy the boundary conditions makes this mathematical requirement moot. For simple cubic stacking of identical spherical pores (Fig. 1a), the critical porosity occurs when $P=0.52$. For other arrangements of spherical pores of uniform size, this transition from closed cells to an interconnected structure occurs at different porosity levels. For orthorhombic stacking (Fig. 1b), the critical porosity is $P=0.60$; for a rhombohedral pore structure (Fig. $1 \mathrm{c}$ ), the critical porosity is $P=0.74$.

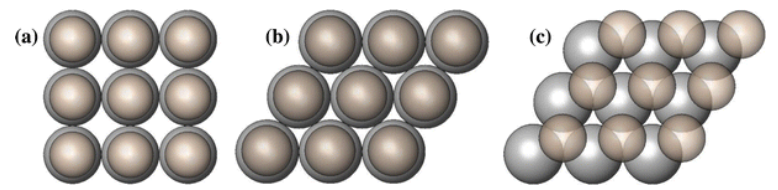

Fig. 1 Regular arrangements of spherical porosity; a cubic, b orthorhombic, and c rhombohedral (top layer of pores intentionally shown smaller for clarity)

For porosity levels beyond the applicable range of the Spriggs (9) equation (i.e., porosity $>40 \%$ ), Rice [38] recognized that the role of pores and matrix material can be reversed leading to a theoretical equation applicable to larger values of porosity:

$E_{\mathrm{p}}=E_{0}[1-\exp (-r\{1-P\})]$ 
where $r$ is empirically determined to have a value of approximately 0.5 . Rice notes that this function is a continuation of the relationship for a simple cubic array of nonintersecting pores in a solid matrix as first derived by Eudier [39] in 1962:

$E_{\mathrm{p}}=E_{0}\left[1-\pi(3 P / 4 \pi)^{2 / 3}\right]=E_{0}\left(1-1.21 P^{2 / 3}\right)$

These two functions are equivalent at $P=\pi / 6 \approx 0.52$ if $r$ is assumed to equal 0.5 . The determination of $r$ is again based on empirical data; the relationship between $m$ in the original Spriggs Eq. (9) and $r$ in Eq. (13) is not defined. The analysis by Eudier is consistent with later studies by Rice [30,31] where he proposes that the ratio of the effective moduli to the solid (non-porous) moduli is directly proportional to the ratio of the minimum contact area to the total cross-sectional area of periodic structures, the basis of the MSA model.

The Eudier Eq. (13) predates similar relationships noted by Martin and Hayes [40] as well as the more recent theoretical work by Herakovich and Baxter [4] who applied the generalized method of cells to study the influence of pore geometry on effective elastic properties. This method uses a "cubical" combination of rectangular subcells to approximate the shape of a spherical void and they conclude that for cubic packing of spherical pores:

$E_{\mathrm{p}}=E_{0}\left(1-1.15 P^{2 / 3}\right)$

The difference in coefficients in Eqs. (13) and (14) is due in part to this cubical approximation.

Analyses by Rice [30] incorporate the MSA models introduced by Knudsen [17] to describe relative strength as a function of volume fraction porosity. Rice concludes that the exponential relationship of Spriggs' Eq. (9), and the analogous Eq. (12) for larger values of porosity, accurately characterizes Young's modulus as a function of volume porosity. Rice maintains that these equations are reasonable approximations of actual models of stacked pores within a matrix, the exponents in these equations have been experimentally determined and that a single parameter, $m$ in Eq. (9) and $r$ in Eq. (12), can be readily correlated with pore character. However, Roberts and Garboczi [41] are critical of the MSA model as this method uses "purely geometrical reasoning to predict the elastic moduli based upon the weakest points within the structure." They maintain that the microstructure that corresponds to the MSA predictions "is not exactly known" and that "MSA models do not provide quantitative agreement" with the moduli of the microstructures studied. They add that while "semiempirical relations generally provide a reasonable means of describing data, extrapolating results, and comparing data among materials... they lack a rigorous connection with microstructure, [and thus] offer neither predictive nor interpretive power." Roberts and Garboczi [41] maintain that Young's modulus is related to the porosity through the empirical equation:

$E_{\mathrm{p}}=E_{0}[1-(P / t)]^{s}(15)$

where the constants $s$ and $t$ are empirically determined parameters (e.g., by fitting simulation results or experimental data) and are dependent on the microstructure of the solid material. With appropriate correlation parameters, their study demonstrates that this equation can be applied to porous ceramics $(P<0.40$ typically) with different morphologies including randomly placed spherical pores, solid spheres, and ellipsoidal pores and may be used as an interpretive tool when the microstructures are unknown.

\section{Additional property-porosity relationships}

Paul [42] notes that the simplest material property-porosity relationship that satisfies the boundary conditions is a special case of the "rule of mixtures" for two-phase solids, which is based on the assumption that both materials contribute to the stiffness of the composite in proportion to their respective moduli and fractional volumes: 
$E_{\mathrm{p}}=E_{1} P+E_{2}(1-P)(16)$

where $E_{1}$ and $E_{2}$ are the Young's moduli of the constituent materials of the composite and $P$ is the volume fraction of material 1. Equation (16) provides an upper bound on the elastic modulus $E_{\mathrm{p}}$ in cases where both constituent materials (phases) have the same Poisson's ratio [42]. When material 1 is a void, then $E_{1}=$ is zero, $P$ becomes the volume porosity and Eq. (16) then reduces to:

$E_{\mathrm{p}}=E_{0}(1-P)(17)$

which is a special case of the Dewey Eq. (1).

Paul also derived an approximate solution for $E_{\mathrm{p}}$ based upon a model of a cube-shaped inclusion when normal uniform stress is applied at the boundary:

$E_{\mathrm{p}}=E_{0}\left(1-P^{2 / 3}\right) /\left(1-P^{2 / 3}+P\right)$

Although empirical results are not quantified in Ref. [42], Paul concludes that this equation "seems to correlate [visually] the experimental data [for cobalt inclusions] quite well." Ishai and Cohen [43] used the same model (i.e., a cubic inclusion within a cubic matrix) with a uniform normal displacement applied at the boundary to obtain:

$E_{\mathrm{p}}=E_{0}\left(1-P^{2 / 3}\right)(19)$

which describes experimental data for porous epoxies with volume porosity less than $30 \%$. They suggest that the approximate lower and upper limits of the effective Young's modulus given by Eqs. (18) and (19), respectively, yield a much narrower band than that given by more rigorous theoretical solutions. Their experimental results indicate that Eq. (19) more closely describes the data than the theoretical solutions of Paul and Hashin [43].

Martin and Haynes [40] further modified this relationship to include a factor $u$, a constant dependent upon "the average void properties of the model (variable for different types of porosity)":

$E_{\mathrm{p}}=E_{0}\left(1-u P^{2 / 3}\right)(20)$

This relationship was "very convincingly confirmed [visually]" with values of $u$ between 1.3 and 2.5 for alumina, bone, and gypsum. However, they acknowledge that empirically derived equations are a "blind alley... in regard to the insight it gives the user..."

Hashin [5] recognized that while the bounds proposed by Paul have the advantage of being exact, they are generally too broadly separated to give a good estimate of the normalized Young's modulus, defined as $E_{\text {porous }} / E_{\text {solid }}$ of most composite materials. Rice [7] along with Boccaccini and Fan [44] attribute to Hashin [5] the equation for the upper bound:

$E_{\mathrm{p}} \leq E_{0}(1-P) /(1+w P)(21)$

where

$w=\left[\left(1+v_{0}\right)\left(13-15 v_{0}\right)\right] / 2\left(7-5 v_{0}\right)(22)$

for the case in which the voids are spherical. Hashin [5] also proposed an equation for a lower bound on the effective modulus but acknowledged that "in such extreme cases as empty cavities,... [these] bounds are not close enough to give a good estimate of the effective moduli" since the gap between the bounds increases with the difference in the elastic properties of the two phases. Hashin [45] notes that these bounds are of practical 
value for a phase stiffness ratio $<10$ and can obviously not provide good estimates for extreme differences in stiffness such as an empty phase (or porous medium). Because the range of the Hashin bounds makes them of limited practical utility for porous solids applications, Ramakrishnan and Arunachalam [46] applied a "correction factor" to the bulk and shear moduli and proposed a relationship for Young's modulus that satisfies "all of the limiting conditions of zero porosity and a totally porous solid". They proposed the following relationship for porous solids with spherical voids distributed randomly:

$E_{\mathrm{p}}=E_{0}(1-P)^{2} /(1+y P)(23)$

where

$y=2-3 v_{0}(24)$

and noted that this equation is restricted "exclusively [to] porous solids and [is not] a special case of two phase materials" [2]. This equation was compared to experimental data for $\mathrm{MgO}, \mathrm{MgAl}_{2} \mathrm{O}_{3}$, and $\mathrm{Sm}_{2} \mathrm{O}_{3}$ with porosity values ranging from 5 to $40 \%$, resulting in discrepancies in predicted moduli of $<20 \%$, comparable to the scatter in the experimental data itself. These data were chosen as these fully dense materials exhibit a broad range of Poisson's ratios ( $v=0.18-0.324$ ). Mondal et al. [47] applied Eq. (23) to closed-cell aluminum foam with porosities ranging from 30 to $80 \%$, demonstrating close agreement with the experimental data over the entire range of porosity with a maximum variation between calculated and experimental results of approximately 10 at $30 \%$ porosity.

Wang and Tseng [1] extended the Hashin Eqs. (21) and (22) by considering the interaction effects between pores. The resulting equations are complicated second-order functions of the bulk modulus, Poisson's ratio, shear modulus, and volume porosity that predicts values for Young's modulus between the upper bound of the Hashin model and the Ramakrishnan model and agrees visually with experimental data up to $40 \%$ porosity.

To assess the effect of pore variables on mechanical properties, Boccaccini [48] derived an equation for the Young's modulus of a porous body from the pore content, shape, and orientation based on an analytical model for composite materials by Mazilu and Ondracek [49]. This equation was then extended to the full porosity range, resulting in the relationship

$E_{\mathrm{p}}=E_{0}\left(1-P^{2 / 3}\right)^{1.21 S}(25)$

where

$S=(z / x)^{1 / 3} \sqrt{1+\left[(z / x)^{-2}-1\right] \cos ^{2} \varphi}(26)$

and $z / x$ is the mean axial ratio (polar or rotational axis/equatorial diameter) of the spheroidal pores, $\varphi$ is the angle between the axes of symmetry of the spheroids and the stress direction; $\cos ^{2} \varphi$ is an orientation factor. The ratio $z / x=1$ for spheres; for oblate spheroids $z / x<1$ and $z / x>1$ for prolate spheroids [49]. Although this equation was proposed to be valid across the entire porosity range, the equation has not been verified experimentally for porosities greater than $40 \%$ [50].

To better compare the various relationships that claim to quantify or predict mechanical properties (e.g., Young's modulus) as a function of porosity, the equations detailed in the preceding paragraphs are summarized in Table 1 and Fig. 2. The normalized modulus $\left(E_{\mathrm{p}} / E_{0}\right)$ versus volume porosity curves depicted in Fig. 2 are based on parameter values noted by the respective researcher with supplemental curves shown for those relationships that specify a range of parameter values. 
Table 1 Summary of relationships for normalized Young's moduli of porous materials $\left(E_{\mathrm{p}} / E_{0}\right)$

\begin{tabular}{|c|c|c|c|c|c|}
\hline General equation form & Researcher & Years & Ref & $\% P$ & Comment \\
\hline \multicolumn{6}{|l|}{ Linear equations } \\
\hline \multirow[t]{5}{*}{$E_{p} / E_{0}=1-a P$} & Dewey & 1947 & {$[6]$} & & \\
\hline & Gatto & 1950 & [7] & & With $a=2.36$ or 2.636 per Rice \\
\hline & Fryxell and Chandler & 1964 & [8] & $2-17$ & With $a \approx 1.9$ \\
\hline & $\begin{array}{l}\text { Hasselman and } \\
\text { Fulrath }\end{array}$ & 1964 & [9] & $<2.5$ & $\begin{array}{l}\text { Attributes calculation of } m \text { to Dewey, MacKenzie and } \\
\text { Hashin; } a=3\left(9+5 v_{0}\right)\left(1-v_{0}\right) /\left[2\left(7-5 v_{0}\right)\right]\end{array}$ \\
\hline & Rossi & 1968 & [11] & $\leq 10$ & $\begin{array}{l}\text { Same as the Hashin eqn at low } \\
\text { porosity; } a=\left(1-v_{0}\right)\left(27+15 v_{0}\right) / \\
{\left[2\left(7-5 v_{0}\right)\right]}\end{array}$ \\
\hline \multicolumn{6}{|l|}{ Power equations } \\
\hline \multirow[t]{5}{*}{$E_{p} / E_{0}=(1-j P)^{i}$} & Bal'shin & 1949 & {$[16]$} & unk & $\begin{array}{l}\text { Only if } j=1 \text { (and } i \text { ranges from } 3 \text { to } 6 \text { ); for strength } \\
\text { not modulus }\end{array}$ \\
\hline & McAdam & 1951 & [18] & $0-40$ & If $j=1$ and $i=3.4$ \\
\hline & Phani et al. & $\begin{array}{l}1986- \\
88\end{array}$ & [19-23] & $5-40$ & $\begin{array}{l}1 \leq j \leq 3.85 \text { for spherical particles; } i \text { ranges from } \\
2 \text { to } 3\end{array}$ \\
\hline & Wagh et al. & 1993 & {$[24]$} & $0-60$ & $\begin{array}{l}j=1 \text {; presents empirical data with } i \text { range from } 2 \text { to } \\
4\end{array}$ \\
\hline & Maitra and Phani & 1994 & {$[25]$} & $0-65$ & Restates Phani et al.; $j=1$ and $i$ ranges from 2 to 4 \\
\hline \multirow[t]{4}{*}{$E_{p} / E_{0}=1-u P^{2 / 3}$} & Eudier & 1962 & [39] & $0-50$ & Only if $u=1.21$ \\
\hline & Ishai and Cohen & 1967 & [43] & $0-30$ & Only if $u=1$ \\
\hline & Martin and Haynes & 1971 & {$[40]$} & $0-50$ & $u$ ranges from 1.3 to 2.5 \\
\hline & $\begin{array}{l}\text { Herakovich and } \\
\text { Baxter }\end{array}$ & 1999 & {$[4]$} & $0-50$ & $u=1.15$ \\
\hline$E_{p} / E_{0}=[1-(P / Q)]^{\mathrm{KQ}}$ & Bert & 1985 & {$[12]$} & $0-20$ & $\begin{array}{l}K \text { is the stress concentration factor }(K=2 \text { for } \\
\text { spheres) and } Q \text { is the maximum porosity possible }\end{array}$ \\
\hline$E_{p} / E_{0}=\left(1-P^{2 / 3}\right)^{1.21 \mathrm{~S}}$ & Boccaccini & $\begin{array}{l}1997 \\
1999\end{array}$ & $\begin{array}{l}{[44]} \\
{[51]}\end{array}$ & $0-40$ & $\begin{array}{l}S=(z / x)^{1 / 3}(1+[(z / \\
\left.\left.x)^{-2}-1\right] \cos 2 \varphi\right)^{1 / 2} \text { where } z / x \text { is the mean axial } \\
\text { ratio (polar axis/equatorial diameter) of the } \\
\text { spheroidal pores }\end{array}$ \\
\hline$E_{p} / E_{0}=\left(1-P^{2 / 3}\right) /\left(1-P^{2 / 3}+P\right)$ & Paul & 1960 & {$[42]$} & - & For inclusions of cubic shape \\
\hline$E_{p} / E_{0}=\left(1-d P+g P^{2}\right)$ & MacKenzie & 1950 & [10] & - & \\
\hline
\end{tabular}




\begin{tabular}{|c|c|c|c|c|c|}
\hline & Chung & 1963 & [13] & - & Cites MacKenzie/Gatto as source for this eqn \\
\hline & Spinner et al. & 1963 & [15] & $0-40$ & $\begin{array}{l}\text { Presents data for } 2.7 \leq d \leq 3.3 \text { and } \\
1.3 \leq g \leq 1.5\end{array}$ \\
\hline$E_{p} / E_{0}=[1-(P / t)]^{S}$ & $\begin{array}{l}\text { Roberts and } \\
\text { Garboczi }\end{array}$ & 2000 & [41] & $0-50$ & $t$ and $s$ are "empirical correlation parameters" \\
\hline \multicolumn{6}{|l|}{ Exponential equations } \\
\hline \multirow[t]{5}{*}{$E_{p} / E_{0}=\exp (-m P)$} & $\begin{array}{l}\text { Duckworth/Ryshkew } \\
\text { itch }\end{array}$ & 1953 & {$[28,29]$} & $0-50$ & $\begin{array}{l}m \text { "has a value of about } 7 \text { for all experimental data on } \\
\text { hand... appears to be independent of pore size"; for } \\
\text { strength not modulus }\end{array}$ \\
\hline & Knudsen & 1959 & [17] & $5-31$ & For strength not modulus \\
\hline & Spriggs & 1961 & {$[32]$} & $0-37$ & For open and closed pores; $m$ ranges from 2.7 to 4.3 \\
\hline & Knudsen & 1962 & [33] & $0-40$ & Presents data to support $m=3.95$ \\
\hline & Rice & 1977 & [7] & $0-40$ & Restates Knudsen \\
\hline$E_{p} / E_{0}=1-\exp (-r\{1-P\})$ & Rice & 1976 & [38] & $>50$ & For pores; $r \approx 0.5$ \\
\hline \multirow[t]{2}{*}{$E_{p} / E_{0}=\exp \left[-\left(n P+q P^{2}\right)\right]$} & Wang, J.C. & 1984 & {$[35,36]$} & $0-32$ & \\
\hline & Panakkal et al. & 1990 & [37] & $0-22$ & \\
\hline \multicolumn{6}{|l|}{ Other equations } \\
\hline \multirow[t]{2}{*}{$E_{p} / E_{0}=(1-P) /(1+w P)$} & Hashin & 1962 & {$[5]$} & - & $\begin{array}{l}w=\left[\left(1+v_{0}\right)\left(13-15 v_{0}\right)\right] / 2\left(7-5 v_{0}\right) \text { for } \\
\text { spherical voids; } w \approx 1.0 \text { for metals }\end{array}$ \\
\hline & Wang, L. and Tseng & 2003 & [1] & $0-50$ & $\begin{array}{l}\text { Same eqn as Hashin if inter-pore interaction is } \\
\text { neglected }\end{array}$ \\
\hline$E_{p} / E_{0}=1+[A P /(1-A P-P)]$ & Hasselman & 1962 & [34] & $0-16$ & $A=$ empirical constant; summarizes data for $A=-4$ \\
\hline \multirow[t]{2}{*}{$E_{p} / E_{0}=(1-P)^{2} /(1+y P)$} & Ramakrishnan & $\begin{array}{l}1990 \\
1993\end{array}$ & $\begin{array}{l}{[46]} \\
{[2]}\end{array}$ & $5-40$ & $y=2-3 v_{0}$ \\
\hline & Mondal et al. & 2007 & [47] & $<80$ & For closed-cell foams \\
\hline
\end{tabular}




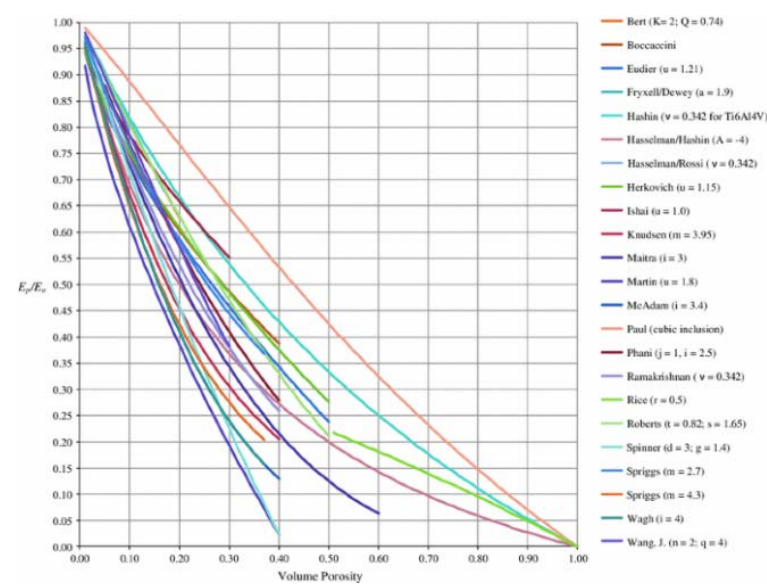

Fig. 2 A graphical comparison of normalized modulus $\left(E_{\mathrm{p}} / E_{0}\right)$ as a function of volume porosity for the various porosity equations (Color figure online)

With the exception of Rossi [11] and Boccaccini [48], the task of detailing and quantifying the influence of pore structure and arrangement within the matrix material on effective moduli has been largely ignored or received scant attention to date.

\section{Conclusions}

Despite the extensive body of research represented by the property-porosity relationships summarized herein, most of these equations are limited in predictive ability. In addition, as noted by Rice, "few investigators studying the dependence of elastic properties on porosity present anything more than density, i.e., average porosity data," when pore shape can significantly alter these relationships and invalidate the results [7]. Moreover, most experimental studies of porous materials do not specify accurate quantitative descriptions of the porosity structure for rigorous validation of theoretical approaches [48]. These limitations adversely affect the utility of a microstructure-property correlation and its ability to predict the property from microstructural measurements for design purposes [51].

Although the development of equations to predict mechanical properties, specifically Young's modulus, of porous materials has been investigated often over the past six decades, there remains a lack of definitive correlation between microstructure of a porous body and the resulting moduli. While these equations provide a means of describing data and comparing results for different materials, the equations are unable to predict moduli to reasonable degree of engineering certainty. Wide disparity in the predicted Young's moduli of porous materials that may result as implicit in each equation are theoretical, analytical, and/or empirical assumptions. The application of these equations to the fabrication of end-use materials and products that incorporate engineered porosity requires a more robust relationship between microstructure, porosity characteristics, and the associated mechanical behavior. With such capabilities currently available, the results of the present review suggest that future research might include: (1) solid modeling of specific non-random ordering of pores of defined size, shape, orientation, and arrangement within a physical construct; (2) subsequent finite element analysis of these solid models that incorporate the predicted engineering moduli; (3) fabrication of physical constructs incorporating various ordered porous microstructures; and (4) experimental testing of the physical constructs with comparison to finite element analyses to assess the accuracy of the predictive equations in capturing changes in the ordered microstructure.

The recent advances in additive manufacturing and the resulting ability to fabricate end-use components which incorporate engineered porosity with deliberate, porous microstructure design require validated predictive equations for Young's modulus over a porosity range of $20-50 \%$ as the relationships presented in the literature 
yield widely disparate results over this range. This review of current data and porosity-property relationships indicates that predictive equations which link pore geometry, orientation, and pore arrangement characterizations with fundamental mechanical properties over a wide porosity range do not yet exist.

\section{References}

1. Wang L, Tseng KK (2003) J Mater Sci 38:3019. doi:10.1023/A:1024736105732

2. Ramakrishnan N, Arunachalam VS (1993) J Am Ceram Soc 76(11):2745

3. O'Kelly KU, Carr AJ, McCormack BAO (2003) J Mater Sci Mater Med 14:379

4. Herakovich CT, Baxter SC (1999) J Mater Sci 34(7):1595. doi:10.1023/A:1004528600213

5. Hashin Z (1962) J Appl Mech 29:143

6. Dewey JM (1947) J Appl Phys 18:578

7. Rice RW (1977) In: MacCrone RK (ed) Properties and microstructure, vol. 11. Academic Press, New York, pp 199-381

8. Fryxell RE, Chandler BA (1964) J Am Ceram Soc 47(6):283

9. Hassselman DPH, Fulrath RM (1964) J Am Ceram Soc 47(1):52

10. Mackenzie JK (1950) Proc Phys Soc Lond Sect B 63:2

11. Rossi RC (1968) J Am Ceram Soc 51(8):433

12. Bert CW (1985) J Mater Sci 20:2220. doi:10.1007/BF01112307

13. Chung D-H (1963) Phil Mag 8:833

14. Wagh AS, Poeppel RB, Singh JP (1991) J Mater Sci 26:3862. doi:10.1007/BF01184983

15. Spinner S, Knudsen FP, Stone L (1963) J Res Natl Bureau Stand C 67C:39

16. Bal'shin MY (1949) Doklady Akademii Nauk SSSR 67(5):831

17. Knudsen FP (1959) J Am Ceram Soc 42(8):376

18. McAdam GD (1951) J Iron Steel Inst 168:346-358

19. Phani KK (1986) J Mater Sci Lett 5:747

20. Phani KK, Niyogi SK (1986) J Mater Sci Lett 5:427

21. Phani KK (1986) Am Ceram Soc Bull 65(12):1584

22. Phani KK, Niyogi SK (1987) J Mater Sci 22(1):257. doi:10.1007/BF01160581

23. Phani KK, Niyogi SK, De AK (1988) J Mater Sci Lett 7:1253

24. Wagh AS, Singh JP, Poeppel RB (1993) J Mater Sci 28:3589. doi:10.1007/BF01159841

25. Maitra AK, Phani KK (1994) J Mater Sci 29:4415. doi:10.1007/BF00376263

26. Paskaramoorthy R, Meguid SA (2000) Int J Solid Struct 37:2341

27. Ji S, Gu Q, Xia B (2006) J Mater Sci 41:1757. doi:10.1007/s10853-006-2871-9

28. Ryshkewitch E (1953) J Am Ceram Soc 36(2):65

29. Duckworth W (1953) J Am Ceram Soc 36(2):68

30. Rice RW (1996) J Mater Sci 31:102. doi:10.1007/BF00355133

31. Rice RW (1996) J Mater Sci 31:1509. doi:10.1007/BF00357860

32. Spriggs RM (1961) J Am Ceram Soc 44:628-629

33. Knudsen FP (1962) J Am Ceram Soc 45(2):94

34. Hassselman DPH (1962) J Am Ceram Soc 45(9):452

35. Wang JC (1984) J Mater Sci 19(3):809. doi:10.1007/BF00540452

36. Wang JC (1984) J Mater Sci 19(3):801. doi:10.1007/BF00540451

37. Panakkal JP, Willems H, Arnold W (1990) J Mater Sci 25:1397. doi:10.1007/BF00585456

38. Rice RW (1976) J Am Ceram Soc 59(11-12):536

39. Eudier M (1962) Powder Metall 9:278

40. Martin RB, Haynes RR (1971) J Am Ceram Soc 54(8):410

41. Roberts AP, Garboczi EJ (2000) J Am Ceram Soc 83(12):3041

42. Paul B (1960) Trans Metall Soc AIME 218:36

43. Ishai O, Cohen $\amalg$ (1967) Int J Mech Sci 9:539

44. Boccaccini AR, Fan Z (1997) Ceram Int 23:239 
45. Hashin Z (1983) Trans ASME 50:481

46. Ramakrishnan N, Arunachalam VS (1990) J Mater Sci 25:3930. doi:10.1007/BF00582462

47. Mondal DP, Ramakrishnan N, Suresh KS, Das S (2007) Scr Mater 57:929

48. Boccaccini AR (1999) J Porous Mater 6:369

49. Mazilu P, Ondracek G (1989) In: Proceedings of Euromech Colloquium, Springer Verlag, Berlin, p 214

50. Personal communication with A.R. Boccaccini, March 13, 2011

51. Boccaccini AR (1994) J Mater Sci Lett 13:1035 